\title{
Liangyi Gao extends lifespan and exerts an antiaging effect in Caenorhabditis elegans by modulating DAF-16/FOXO
}

\author{
Liling Zeng • Chen Sun - Zhong Pei - Tianchan Yun - Shaoyi Fan • \\ Simei Long $\cdot$ Tengteng Wu $\cdot$ Ziwen Chen $\cdot$ Zhimin Yang $\cdot$ Fuping Xu
}

Received: 2 April 2019/Accepted: 10 July 2019/Published online: 22 July 2019

(C) The Author(s) 2019

\begin{abstract}
Liangyi Gao (LYG), a traditional Chinese medicine, is composed of Ginseng and Radix Rehmanniae Preparata, both of which have been shown to have antiaging properties. In Eastern countries, LYG is used to delay functional declines related to aging and has an obvious antiaging effect in clinical practice. However, little data from evidence-based medicine is available regarding whether LYG is beneficial overall, particularly with respect to lifespan, and how LYG functions. To address these issues, Caenorhabditis
\end{abstract}

\author{
L. Zeng $\cdot$ T. Yun $\cdot$ S. Fan $\cdot$ Z. Chen \\ The Second Clinical College of Guangzhou University of \\ Chinese Medicine, Guangzhou, China \\ e-mail: liling.zeng@yale.edu \\ T. Yun \\ e-mail: yuntc92@163.com \\ S. Fan \\ e-mail: 408671469@qq.com \\ Z. Chen \\ e-mail: 651061776@qq.com \\ C. Sun $\cdot$ Z. Yang $(\bowtie) \cdot$ F. Xu $(\bowtie)$ \\ Guangdong Provincial Hospital of Chinese Medicine, 111 \\ Da De Rd, Yuexiu District, \\ Guangzhou 510120, Guangdong Province, China \\ e-mail: yangyo@vip.tom.com \\ C. Sun \\ e-mail: sunchen87319@163.com \\ F. Xu \\ e-mail: xufuping163@163.com
}

elegans, a useful organism for such studies, was employed to explore the antiaging effect and mechanism of LYG in this study. The results showed that LYG could obviously extend lifespan and slow agingrelated declines in N2 wild-type C. elegans. To further characterize these antiaging effects and stress resistance, reproductive tests and other aging-related tests were performed. We found that $\mathrm{LYG}$ enhanced resistance against oxidative and thermal stress, reproduction, pharynx pumping, motility and growth in N2

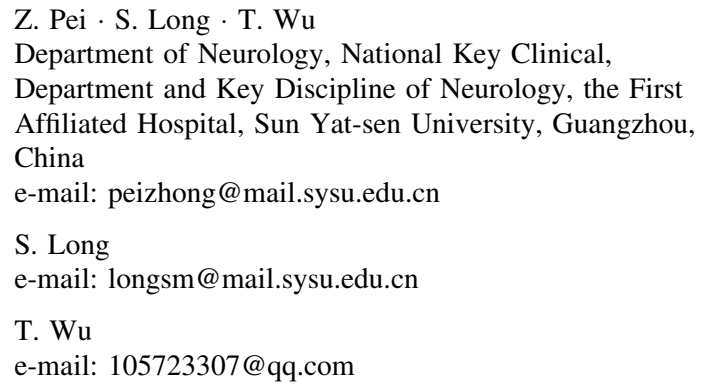


wild-type $C$. elegans. In addition, we analyzed the mechanism for these effects by measuring the activity of superoxide dismutase (SOD) and the expression levels of aging-related genes. We found that LYG enhanced the activities of antioxidant enzymes and upregulated the genes daf-16, sod-3 and sir-2.1, which mediated stress resistance and longevity. In conclusion, LYG had robust and reproducible life-prolonging and antiaging benefits in C. elegans via DAF-16/ FOXO regulation.

Keywords Antiaging - Liangyi Gao - Traditional Chinese Medicine $\cdot$ Caenorhabditis elegans $\cdot$ DAF-16/ FOXO regulation

\section{Introduction}

Aging is a complex process characterized by the progressive failure of maintenance and repair pathways. Aging leads to dysfunction, disease, and ultimately, death, which has long been a concern (Blagosklonny and Hall 2009). Although people have attempted to explore the mechanisms of aging, these mechanisms are complicated, and a specific antiaging intervention is still not known (Chung et al. 2011; Mikhelson and Gamaley 2012; Chen et al. 2013; Isobe 2016).

As a complementary medicine, traditional Chinese medicine (TCM) has 5000 years of history and has attracted much attention for its medical efficacy as well as its preventative functions (Zhou 1982; Chen 2015; Liu et al. 2017). Recently, numerous studies have indicated that many TCMs have an array of antiaging effects (Liang and Yin 2010; Wan et al. 2014; Hsu et al. 2015). Liangyi Gao (LYG) is a TCM formula described in Jing Yue's Complete Work, which is mainly composed of 2 ingredients, Panax Ginseng and Radix Rehmanniae Preparata. Ginseng combined with Rehmannia glutinosa is a classic Chinese herbal prescription that has invigorates Qi and nourishes blood; this prescription is now commonly used to treat weakness or chronic debilitating diseases. Both Ginseng and Rehmannia glutinosa have been demonstrated to have antiaging effects in previous studies (Yang et al. 2017; Bai et al. 2018). In addition, ginsenoside $\mathrm{Rg} 1$, an active ingredient in Ginseng, has effects that include antiaging as well as improving immunity (Cheng et al. 2005; Zhou et al. 2011; Chen et al. 2014; Tang et al. 2015; Zhou et al. 2015); additionally, catalpol, an active ingredient in Rehmannia glutinosa, also exhibits antiaging effects (Zhang et al. 2013; Seo et al. 2015). Moreover, we have drawn from more than 10 years of clinical experience, which has shown that LYG has strong antiaging effects. Since little data from evidencebased medicine is available about whether LYG is beneficial overall, we performed this study to confirm its benefits and to explore the mechanism of its antiaging effects using $C$. elegans, which has become a widely accepted model for aging research because of its short lifespan, morphology, ease of culture, and ease of genetic manipulation (Guarente and Kenyon 2000; Wang et al. 2014). Moreover, since C. elegans shares nearly two-thirds of its genes with humans, it is used as a well-characterized experimental system for aging and aging-associated disease research (Sluder and Baumeister 2004).

To investigate whether LYG has an antiaging effect, we performed lifespan assays and stress resistance assays, and we assessed other aging-related properties with wild-type C. elegans N2 (Bristol). Furthermore, to examine the underlying mechanisms, we analyzed the effects of LYG on the activity of superoxide dismutase (SOD) and on the expression levels of aging-related genes.

\section{Methods}

Drug preparation

The LYG formula contains a 1:2 ratio of Ginseng and Radix Rehmanniae Preparata. The formula was extracted by water, and its concentration was determined by the amount of the crude drug in solution. The Ginseng and Radix Rehmanniae Preparata were provided by Kangmei Pharmaceutical (Guangzhou, China), and the LYG was produced according to the detailed specifications of Jing Yue's Complete Work. Ginsenoside Rg1 (Lot: A0503AS) and catalpol (Lot: 50728AS) were provided by Meilunbio (Dalian, China). All drugs were dissolved in dimethylsulfoxide (DMSO) and were diluted in E. coli OP50 solution when the drugs were added during the preparation of agar plates. Detailed information about the drugs used in the different groups is shown in Table 1. 
Table 1 The components, dose proportions, and original concentrations of the drugs in the different groups

\begin{tabular}{llll}
\hline Groups and drugs & Components & Dose proportion & ${\text { Original concentration }(\mathrm{mg} / \mathrm{ml})^{\mathrm{a}}}^{\mathrm{a}}$ \\
\hline LYG & Ginseng and Radix Rehmanniae Preparata & $1: 2$ & $1 \mathrm{mg} / \mathrm{ml}$ \\
Rg1 & Ginsenoside Rg1 & - & $150 \mu \mathrm{g} / \mathrm{ml}$ \\
Catalpol & Catalpol & - & $150 \mu \mathrm{g} / \mathrm{ml}$ \\
Rg1 + Catalpol & Ginsenoside Rg and catalpol & $1: 1$ & $150 \mu \mathrm{g} / \mathrm{ml}$ \\
Control & DMSO & - & Equal to the other groups \\
\hline
\end{tabular}

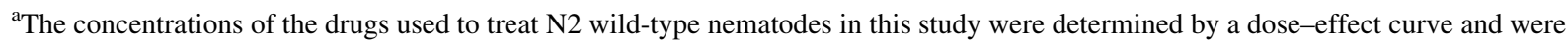
chosen based on the dose with the greatest effect on the lifespan of N2 wild-type nematodes; DMSO, dimethylsulfoxide

Caenorhabditis elegans: strains and maintenance

The Genetics Center (CGC) at the University of Minnesota (Minneapolis, MN, USA) provided wildtype C. elegans N2 (Bristol), the CF1553 C. elegans strain, TJ356 C. elegans strain, and E. coli OP50. $C$. elegans strains were maintained at $20{ }^{\circ} \mathrm{C}$ on solid nematode growth medium (NGM) plates seeded with E. coli OP50.

\section{Lifespan analysis}

Age-synchronized N2 nematodes were transferred to NGM plates containing drugs or vehicle control (DMSO). Two NGM plates containing 25 worms per plate were established for each group, and the worms were transferred to a new NGM plate every day during the first 7 days to prevent new eggs from causing a disturbance. The survival rate was assessed every other day until death. The nematodes were considered dead when they failed to respond to touch using a platinum loop (touch-provoked method). The lifespan assay was repeated in more than two independent trials.

\section{Assessment of stress resistance}

Age-synchronized N2 worms were bred on NGM plates with drugs or vehicle control (DMSO). For the heat tolerance assay, the adult day 4 worms $(n=50)$ were transferred to fresh plates and then incubated at $37{ }^{\circ} \mathrm{C}$. Survival was recorded every hour until all worms died. Oxidative stress tolerance was assessed as described previously with minor modifications (Munoz and Riddle 2003). Briefly, the adult day 4 worms $(n=50)$ were placed on plates containing various concentrations of hydrogen peroxide (from $0 \mathrm{mM}$ to $1 \mathrm{mM}$, in intervals of $0.2 \mathrm{mM}$ ), and then survival was recorded over $15 \mathrm{~h}$. The survival of worms was determined by touch-provoked movement, as described above. Each test was performed at least two times.

\section{Measurement of SOD activity}

To assess the antioxidant enzyme activity, worm homogenates were prepared. Briefly, wild-type worms $(\mathrm{n}=50)$ were collected from plates with M9 buffer on adult day 5 and were washed 3 times. Then, the collected worms were resuspended in homogenization buffer (10 mM Tris-HCl, $150 \mathrm{mM} \mathrm{NaCl}$, and $0.1 \mathrm{mM}$ EDTA, $\mathrm{pH}$ 7.5) and homogenized with an ultrasonic wave method on ice. SOD activity was spectrophotometrically measured by analyzing the decolorization of formazan that results from an enzymatic reaction between xanthine and xanthine oxidase. A Total Superoxide Dismutase (T-SOD) Assay Kit (Hydroxylamine method) and a Total Protein Assay Kit (with standards: BCA method) were used for the determination of the protein concentration and SOD activity, respectively, and were purchased from Nanjing Jiancheng Bioengineering Institute (Nianjing, China). The procedure was performed in strict accordance with the manufacturer's protocol.

\section{Measurement of aging-related factors}

Age-synchronized N2 worms were bred on NGM agar plates with or without drugs. On the fourth day of adulthood, single worms were transferred to fresh plates, and their pharynx contractions were counted under an inverted microscope for $10 \mathrm{~s}(\mathrm{n}=10)$. For 
the reproduction assay, 5 worms were raised from embryos. L4 larvae were individually transferred to a fresh plate every day to distinguish the parent from the progeny. The progeny was counted at the L2 or L3 stage. For the growth alteration assay, photographs were taken of adult day 4 worms, and the body length of each animal was analyzed by Nikon software (Nikon, Japan). For the body movement assay, on the seventh day of adulthood, single worms were transferred to fresh plates, and their body movements were recorded under an inverted microscope for $20 \mathrm{~s}$. The body movements of the animals were analyzed by Nikon image software, and the data are expressed as the total distance travelled. All the tests were repeated more than two times. Lipofuscin, an endogenous marker of cellular damage during aging (Brunk and Terman 2002), was also assessed on the 10th day of adulthood, and the autofluorescence level of lipofuscin was observed under a fluorescence microscope. The fluorescence intensity was quantified using ImageJ software.

Fluorescence microscopy and visualization

Age-synchronized transgenic nematodes, including the CF1553 strain that contained a SOD-3::GFP reporter and the TJ356 strain that contained a daf$16::$ GFP reporter, were maintained in the presence or absence of LYG. On the third day of adulthood, the nematodes were exposed to heat shock at $37{ }^{\circ} \mathrm{C}$ for $2 \mathrm{~h}$ and allowed to recover at $20^{\circ} \mathrm{C}$ for $4 \mathrm{~h}$. Prior to microscopic observation, transgenic animals were anesthetized with sodium azide (2\%) and mounted on a $2 \%$ agarose pad. The GFP fluorescence of GFPexpressing populations was directly observed under a fluorescence microscope (Nikon, Japan). To determine the protein expression levels, photographs of the transgenic worms were taken and analyzed using ImageJ software. All experiments were performed in triplicate.

Quantitative analysis of aging-related genes in C. elegans

Synchronized C. elegans were treated with or without LYG for 3 days at $20{ }^{\circ} \mathrm{C}$. The worms were collected, washed three times with M9 buffer, transferred to $1.5 \mathrm{ml}$ RNase-free microfuge tubes, and pelleted by centrifugation at $3000 \mathrm{rpm}$ for $1 \mathrm{~min}$. For every sample containing approximately 600 worms, $1 \mathrm{ml}$ TRIzol reagent was added (TaKaRa, Beijing, China); the samples were vortexed and then fully homogenized. For total RNA extraction, $200 \mathrm{ml}$ of chloroform was added, and the worm suspension was vigorously shaken and centrifuged at $12,000 \times g$ for $15 \mathrm{~min}$. The total nematode RNA in the supernatant was isolated using isopropanol and washed with $75 \%$ ethanol. The RNA concentration was quantified using a Nanodrop spectrophotometer. Complementary DNA was produced using random 6-mer and oligo (dT) primers (TaKaRa, Beijing, China) according to the manufacturer's protocol. Quantitative real-time polymerase chain reaction (qPCR) was performed, and SYBR green (TaKaRa, Beijing, China) was used as the detection method. Act-1 was used as a reference gene, and the expression levels of each mRNA relative to the act-1 gene were calculated with the comparative $2^{-\Delta \Delta C T}$ method. The experiment was repeated in triplicate.

\section{Statistical analyses}

GraphPad Prism 6.0 was used for statistical analyses. For the lifespan assay, Kaplan-Meier survival analysis was conducted, and $P$ values were calculated using the log-rank test. A Student's $t$ test was performed to compare two datasets. All results are expressed as the mean \pm the standard error of the mean (SEM). Values of $P<0.05$ were considered significant.

\section{Results}

Effect on lifespan extension and stress resistance

To evaluate the lifespan-extending properties of LYG, lifespan assays were performed using wild-type worms. Herein, we found that worms exposed to LYG showed a higher rate of increase in lifespan than the worms exposed to $\operatorname{Rg} 1$, catalpol, or the control; additionally, the $\operatorname{Rg} 1$ and catalpol combination treatment showed a benefit similar to that of $\mathrm{LYG}$ treatment (shown in Fig. 1a and Table 2). To evaluate stress resistance, we performed heat stress assays and oxidative stress assays. As shown in Fig. 1b, compared to the worms in the control group, worms fed $1 \mathrm{mg} / \mathrm{ml} \mathrm{LYG} \mathrm{had} \mathrm{a} \mathrm{significantly} \mathrm{increased} \mathrm{mean}$ lifespan during heat stress $(P<0.01)$. Resistance to 

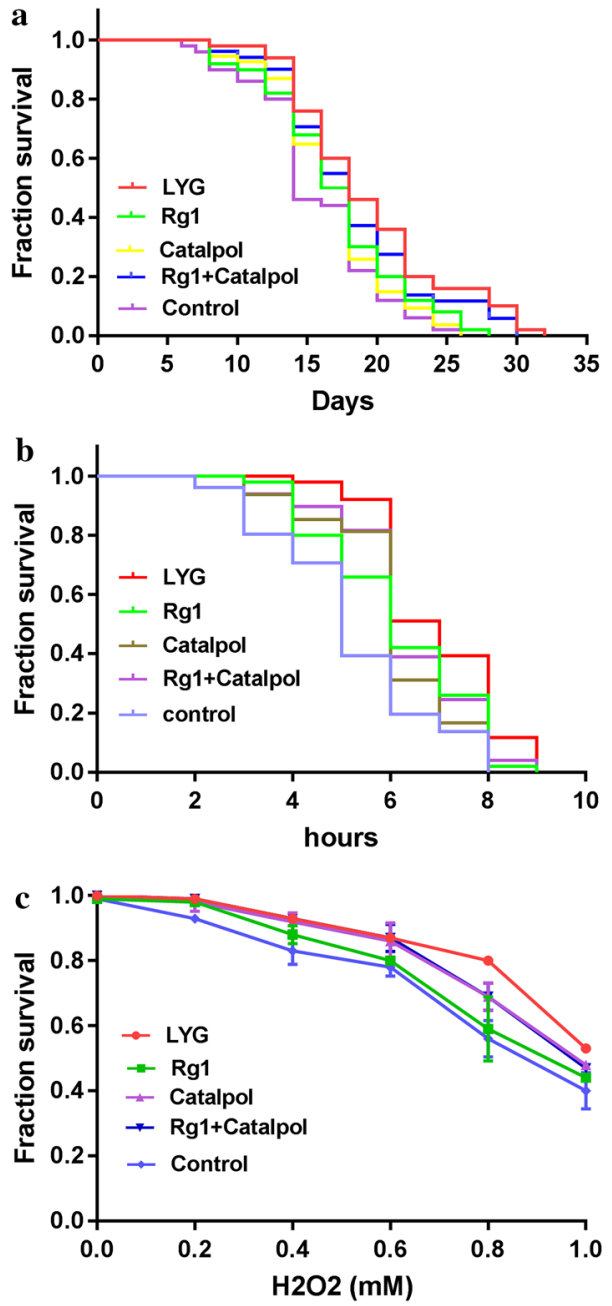

Fig. 1 Effect on the lifespan and stress resistance in C. elegans N2. a Effect on lifespan extension. b Effect on heat stress resistance. $\mathbf{c}$ Effect on oxidative stress resistance oxidative stress was examined by exposing animals to hydrogen peroxide, an intracellular free radical-generating compound. The results also showed that LYGtreated wild-type worms lived longer than the control worms during hydrogen-peroxide-induced oxidative stress (Fig. 1c, $P<0.05$ ).

\section{Effect on aging-related factors}

Previous studies have suggested that longevity is closely connected with reproduction, pharynx pumping, body size and motility in many species, including in C. elegans (Zhang et al. 2013). In this study, we showed that LYG treatment, as well as the $\operatorname{Rg} 1$ and catalpol combination treatment, significantly increased the total progeny number compared to that in the control group (shown in Fig. 2 a, $P<0.05$ ). It was especially interesting that the LYG treatment delayed the decline in reproduction and significantly increased the progeny number during the later stage of reproduction (shown in Fig. 2a, during days 4 and 5), suggesting that LYG treatment could delay reproductive decline. In addition, a small but significant change in the body length of worms was detected after LYG exposure (shown in Fig. $2 \mathrm{~b}, P<0.05$ ), suggesting that the antiaging effect of LYG is dependent on growth as well as on fertility. Then, we measured the rate of pharyngeal pumping to estimate the muscle activity and the motor ability of the worms. As shown in Fig. 2c, the rate of pharyngeal contractions gradually declined with increasing age, and this ageassociated decline was delayed by LYG treatment. Furthermore, we evaluated whether LYG affected

Table 2 Effect on lifespan extension

\begin{tabular}{lllll}
\hline & Mean lifespan $^{\mathrm{a}}$ & Maximum lifespan & ${\text { Decline in mean lifespan }(\%)^{\mathrm{b}}}^{\mathrm{b}}$ & - \\
\hline LYG & $19.44 \pm 0.80$ & 32 & - & $0.03^{*}$ \\
Rg1 & $17.08 \pm 0.69$ & 28 & 12.1 & $0.00^{* * *}$ \\
Catalpol & $16.85 \pm 0.57$ & 26 & 2.3 & 0.29 \\
Rg1 + Catalpol & $18.27 \pm 0.75$ & 30 & 6.0 & $0.00^{* * *}$ \\
Control & $15.7 \pm 0.64$ & 26 & 19.2 & \\
\hline
\end{tabular}

${ }^{a}$ Mean lifespan is presented as the mean \pm SEM

${ }^{\mathrm{b}}$ Decline in mean lifespan compared with the LYG group (\%)

${ }^{\mathrm{c}}$ Statistical significance of the difference between the survival curves was determined by the log-rank test using Kaplan-Meier survival analysis. Differences compared to the LYG group were considered significant at $* P<0.05$ and $* * * P<0.01$ 

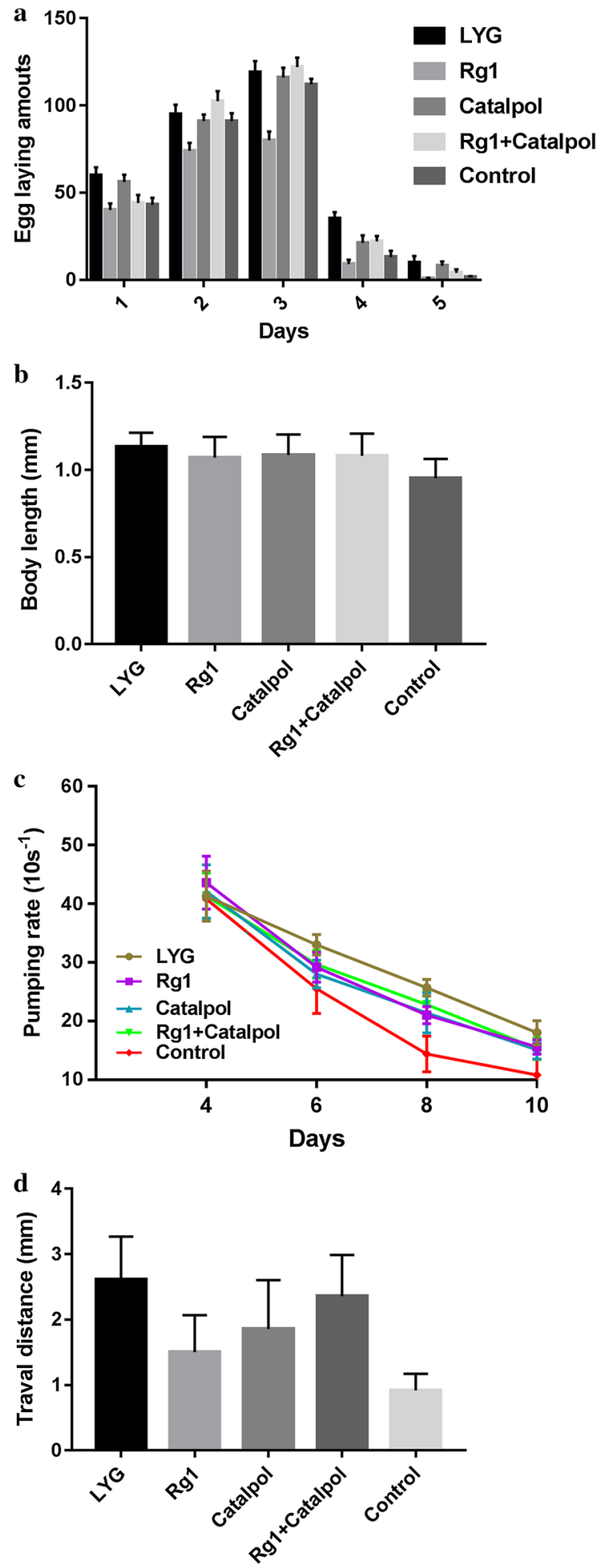

Fig. 2 Effects on aging-related factors. a Effect on reproduction. b Effect on body length. $\mathbf{c}$ Effect on pharyngeal pumping. d Effect on body movement age-associated changes in $C$. elegans, such as body movements. To estimate the health span of worms, we recorded the distance traveled in $20 \mathrm{~s}$. As shown in Fig. 2d, compared to the control group, the LYG group exhibited a significant increase in body movement $(P<0.01)$, suggesting that the functional aging of worms is strongly delayed by LYG. We measured the autofluorescence level of lipofuscin, and the results revealed that compared to control worms, the LYGtreated worms exhibited significantly attenuated fluorescence intensity from intestinal lipofuscin $(34.20 \%$ decrease; $P<0.001$, Fig. 3).

Mechanism of LYG treatment effects on the lifespan of C. elegans

\section{Effect of LYG on antioxidant enzyme activity}

To verify the possible mechanism of LYG-mediated lifespan extension and stress tolerance elevation, the activities of stress resistance proteins were investigated in wild-type worms. In the present study, we measured the activities of antioxidant enzymes such as SOD using prepared worm homogenates. As noted in Fig. 4a, compared to that in the control group, SOD was significantly upregulated in the $1 \mathrm{mg} / \mathrm{ml} \mathrm{LYG}$ group $(P<0.05)$. Next, we verified that SOD was upregulated due to the upregulation of the sod-3 gene. Therefore, we conducted another assay with CF1553 (muIs84 [(pAD76) sod-3p::GFP + rol-6 (su1006)]); during heat stress, GFP (sod-3p::GFP) will be expressed in the head and tail and around the vulva. As shown in Fig. 4b-d, the fluorescence intensity that resulted from SOD-3::GFP expression in LYG-treated CF1553 worms was stronger than that in the controltreated worms $(P<0.01)$.

Effects of LYG treatment on the expression of agingrelated genes

LYG treatment significantly increased the expression levels of the daf-16, sod-3 and sir-2.1 genes $(P<0.05$, shown in Fig. 5a). To verify that the expression of the daf-16 gene was activated, we conducted another assay with the TJ356 strain (zIs356[daf-16p::daf-16a/ $\mathrm{b}::$ GFP + rol-6]), which shows nuclear localization of daf-16 during heat stress, as indicated by GFP (daf$16::$ GFP) expression. To induce the nuclear localization of daf-16, worms were incubated at $36{ }^{\circ} \mathrm{C}$ for $2 \mathrm{~h}$ 

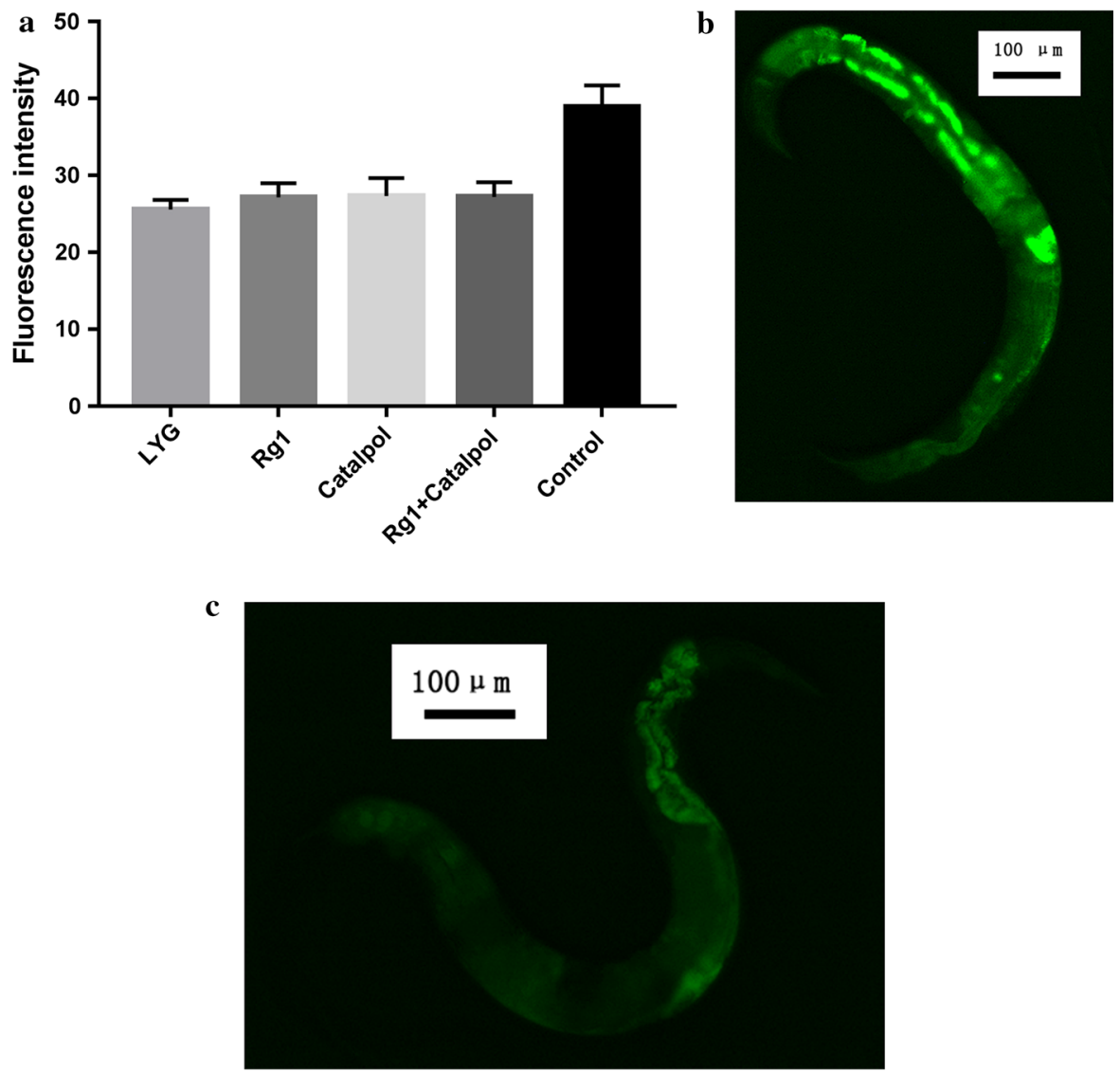

Fig. 3 Effect on lipofuscin accumulation. a The lipofuscin accumulation was lowest in LYG-treated worms, and the difference compared to the control-treated worms was

to induce heat shock. The worms were subjected to GFP expression analysis on the fourth day of adulthood. As shown in Fig. 5b-d, after the worms experienced heat shock, they showed the nuclear localization of daf-16. Similarly, the worms treated with LYG also showed the nuclear localization of daf16 , but the control worms did not show a change in daf-16 localization.

\section{Discussion}

Restoratives in traditional Chinese drugs, such as Ginseng and Radix Rehmanniae Preparata, can attenuate age-related declines, as measured by several physiological and functional indices (Lee et al. 2007; Ko et al. 2010; Wan et al. 2014). Using the short-lived nematode $C$. elegans, we established a genetic system significant $(P<0.001)$. b Fluorescence intensity of intestinal lipofuscin in the control worms. c Fluorescence intensity of intestinal lipofuscin in the LYG-fed worms

to examine the effects and the mechanism of LYG on longevity and aging. This work shows that treatment with LYG caused the extension of the mean adult lifespan as well as improved physiological function and stress tolerance. Finally, we showed that the beneficial effects of LYG treatment appear to require the activity of DAF-16/FOXO signaling, which also regulate stress resistance.

In modern times, an increasing number of agerelated diseases, such as chronic degenerative diseases and other aging-related dysfunctions, have threatened human health (Gladyshev and Gladyshev 2016; Krut'ko et al. 2018). Contrary to mainstream modern medicine, TCM aims to interfere with the aging process as early as possible, thus preventing and delaying the occurrence and development of agerelated diseases; this approach has begun to attract increasing attention from researchers (Liang and Yin 
Fig. 4 a Effect of LYG on antioxidant enzyme (SOD) activity. b The fluorescence intensity of SOD-3::GFP expression in LYG-treated CF1553 worms was significantly stronger than that in the worms in the control group.

c Fluorescence intensity of SOD-3::GFP expression in LYG-treated CF1553 worms. d Fluorescence intensity of SOD-3::GFP expression in control CF1553 worms

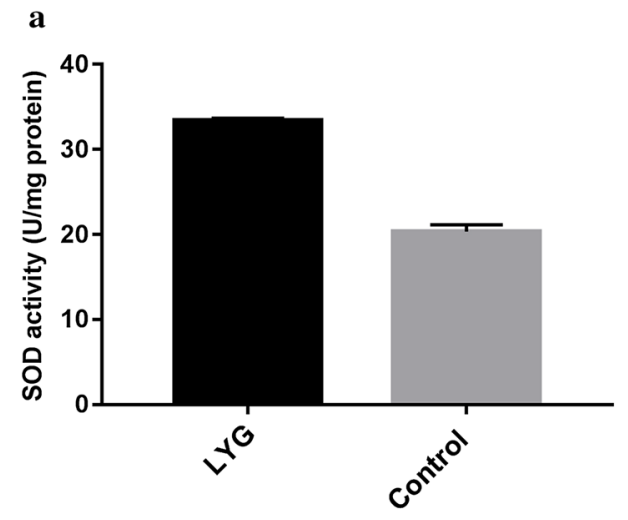

c

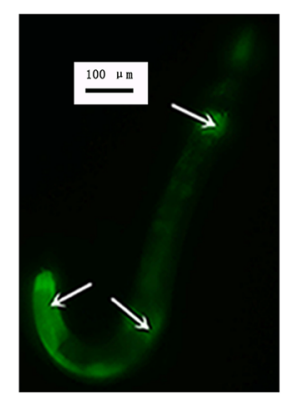

b

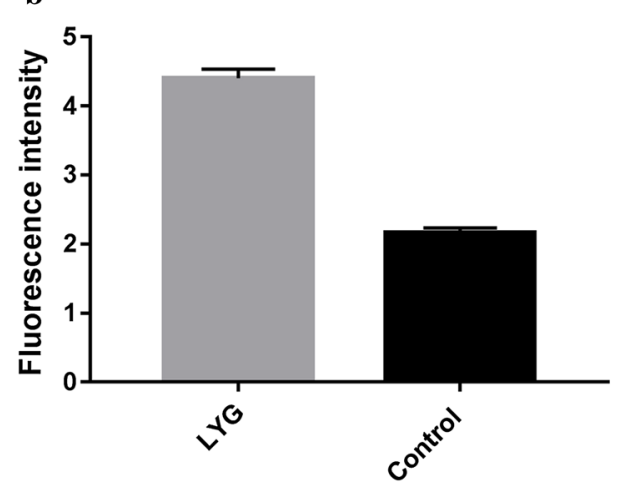

d

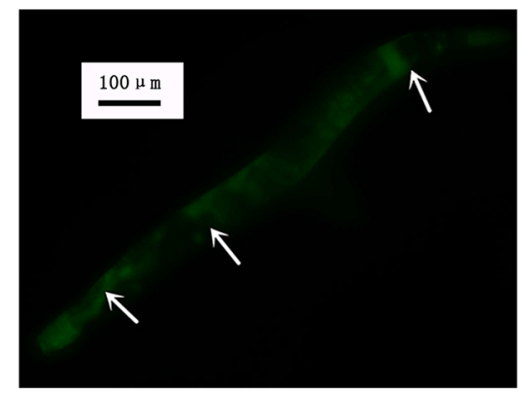

2010; Chen 2015). Moreover, many recent studies have focused on developing new products using herbal plants or compounds derived from these plants, which are considered to have low toxicity and to be free of adverse effects. For a long time, mixtures of medicinal plants have been used as traditional prescriptions in Eastern countries because the compounds in these mixtures have the potential to complement and enhance the effects of one another. According to TCM theory, LYG has reinforces Qi and nourishes Yin, as well as detoxifies the kidney and spleen, which are the main treatment strategies for aging (Hu 1986; Shen et al. 2002). Previous studies have shown that Ginseng and Radix Rehmanniae Preparata have antiaging effects; the active ingredients ginsenoside Rg1 (from Ginseng) and catalpol (from Radix Rehmanniae Preparata) can also exert antiaging effects (Chen et al. 2008; Zhang et al. 2008; Li et al. 2016). LYG is composed of Ginseng and Radix Rehmanniae Preparata and has been clinically proven to be effective, but further research is needed on the nature of LYG due to the complexity of its composition.
In this study, we explored the antiaging activity of LYG using a $C$. elegans model. We found that LYG treatment significantly prolonged the lifespan of wildtype worms under normal and stress conditions. This result indicates that LYG probably has good antiaging effects and preventative effects on age-related diseases. In addition, LYG treatment showed higher lifeprolonging effects than its active ingredients ginsenoside Rg1 or catalpol (shown in Table 2), suggesting that the synergy of Ginseng and Radix Rehmanniae Preparata can lead to a stronger antiaging effect. Currently, the goal of antiaging medicine has shifted from simply extending lifespan to increasing health span. In this study, we showed that the agerelated decline in functions, such as pharyngeal pumping, body movement, reproductive capacity and the reduction of lipofuscin accumulation, were effectively delayed in LYG-treated worms compared to control-treated worms, indicating that LYG could enhance the health span of worms.

To understand the underlying mechanisms by which LYG exerts antiaging effects, C. elegans was employed as an in vivo model, and SOD activity and 
Fig. 5 a The quantified relative expression of mRNA. b Effects of heat shock on the nuclear localization of daf-16. c Effects of LYG treatment on the nuclear localization of daf-16. d The control group
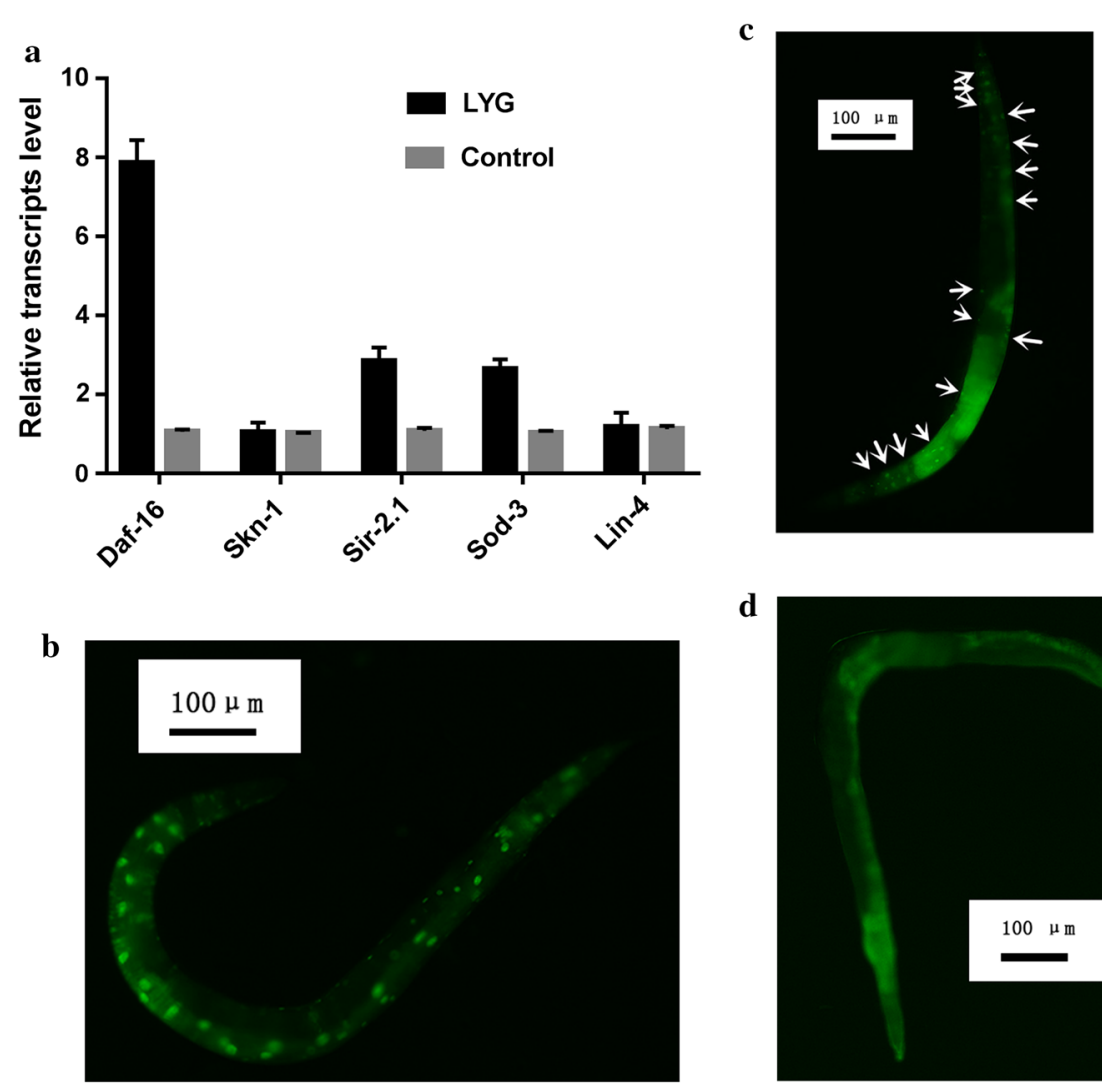

d

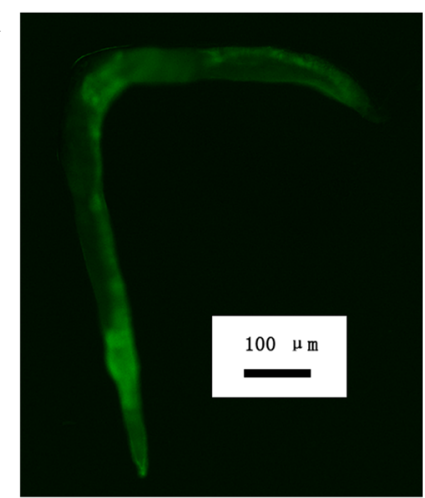

the expression levels of aging-related genes were evaluated. Previous studies have suggested that the accumulation of oxidative stress caused oxygen free radicals and that this was a major factor in aging; thus, enhancing oxidative stress resistance could delay aging (Finkel and Holbrook 2000; Bokov et al. 2004). Our research indicated that LYG-induced the elevation of antioxidant enzyme activity, such as SOD activity, resulting in the elimination of oxygen free radicals, which might have antiaging effects. Previous studies have revealed that gene expression can change during aging in C. elegans (Murphy et al. 2003). LYG treatment might improve survival by activating the genes involved in the stress response, which affects lifespan in C. elegans. As shown in Fig. 4b-d, the fluorescence intensity of SOD-3::GFP expression in LYG-treated CF1553 worms was stronger than that in control worms, which suggests that LYG treatment increases the activity of SOD, probably via the activation of the sod-3 gene. Interestingly, a previous study suggested a possible causal association between antioxidant activities and longevity and a possible mechanism to account for the hormetic effects (Kitani et al. 2002; Calabrese et al. 2015). The daf-16 gene belongs to the "vitagenes family", which is a group of genes strictly involved in preserving cellular homeostasis during stressful conditions (Calabrese et al. 2007); the antiaging effect of LYG could occur via a mechanism that involves the hormetic stimulation of a vitagene pathway. LYG may induce an adaptive stress response and activate adaptive cellular stress response pathways, such as Forkhead box protein (FOXO) transcription factors (daf-16 in Caenorhabditis elegans), which induce the expression of FOXO target genes (such as sod-3) that encode antioxidant enzymes (SOD) (Mattson 2008; Calabrese et al. 2011).

Furthermore, using qRT-PCR, we confirmed that the expression of the antiaging genes daf-16, sir-2.1 and sod-3 were upregulated in LYG-treated worms. LYG treatment might improve survival by activating the genes involved in stress responses that affect lifespan in C. elegans. The two transcription factors daf-16 and sod-3 can promote the expression of antioxidant or detoxification enzymes in C. elegans. 
The DAF-16/FOXO transcription factors promoted the expression of genes that confer extended longevity and enhanced stress resistance (Mukhopadhyay et al. 2006; Chavez et al. 2007). Sod-3, which is the downstream target of daf-16, promotes the expression of detoxification enzymes, such as SOD, in response to oxidative stress (Honda et al. 2010; Zhao et al. 2017). LYG treatment significantly increased the expression levels of the daf-16 and sod3 genes (Fig. 5a), further verifying that LYG regulated sod-3 downstream of daf-16 to extend lifespan and increase stress resistance. LYG treatment also significantly upregulated the expression level of sir 2.1 (shown in Fig. 5a), suggesting that LYG treatment can increase $C$. elegans lifespan through sir-2.1, which interacts with 14-3-3 proteins to activate daf-16 and extend lifespan (Berdichevsky et al. 2006). Sir-2.1 is another evolutionarily conserved regulator of longevity that increases the lifespan of $C$. elegans through either the downregulation of the insulin/insulin growthfactor signaling (IIS) pathway or the direct activation of daf-16 in parallel to IIS signaling. Furthermore, we conducted a nuclear localization assay with the TJ356 strain to further verify whether the daf-16 gene was activated. As shown in Fig. 5b-d, after experiencing heat shock, the worms showed the nuclear localization of daf-16. Similarly, the worms treated with LYG also showed the nuclear localization of daf-16, suggesting that LYG treatment exerted a life-prolonging and antiaging effect in $C$. elegans by activating the gene daf-16.

\section{Conclusions}

In conclusion, this study demonstrates that LYG, a TCM formulation, increases stress resistance and promotes longevity in C. elegans via DAF-16/FOXO regulation.

Acknowledgements This study was conducted at the Key Laboratory of Neurology Department, The First Affiliated Hospital, Sun Yat-sen University for Model Organisms, and Lingnan Medical Research Center of Guangzhou University of Chinese Medicine, Guangzhou, China. The authors are grateful to Professor Qiuying $\mathrm{Xu}$ and Dr. Fengyin Liang for their technical support and assistance.

Author contributions Liling Zeng, Fuping $\mathrm{Xu}$ and Zhimin Yang conceived and designed the study. Liling Zeng, Tengteng Wu, Simei Long, Ziwen Chen, Chen Sun, and Shaoyi Fan performed the experiments. Liling Zeng and Chen Sun analyzed the data. Liling Zeng, Chen Sun, Zhong Pei and Tianchan Yun wrote the first draft of the manuscript. All authors searched and reviewed the literature. All authors read and approved the final manuscript.

Funding This study was funded by the Guangdong Hospital of Traditional Chinese Medicine Scientific and Technological of China (Grant Number YN2015QN08) and the Natural Science Foundation of China (Grant Number 81503515).

Availability of data and materials All the data are presented in the manuscript, and the authors declare that the submitted manuscript does not contain previously published data.

\section{Compliance with ethical standards}

Conflict of interest The authors declare that they have no conflict of interest.

Open Access This article is distributed under the terms of the Creative Commons Attribution 4.0 International License (http:// creativecommons.org/licenses/by/4.0/), which permits unrestricted use, distribution, and reproduction in any medium, provided you give appropriate credit to the original author(s) and the source, provide a link to the Creative Commons license, and indicate if changes were made.

\section{References}

Bai L, Shi GY, Yang YJ, Chen W, Zhang LF, Qin C (2018) Rehmannia glutinosa exhibits anti-aging effect through maintaining the quiescence and decreasing the senescence of hematopoietic stem cells. Anim Model Exp Med $1: 194-202$

Berdichevsky A, Viswanathan M, Horvitz HR, Guarente L (2006) C. elegans SIR-2.1 interacts with 14-3-3 proteins to activate DAF-16 and extend life span. Cell 125:1165-1177

Blagosklonny MV, Hall MN (2009) Growth and aging: a common molecular mechanism. Aging (Albany NY) $1: 357-362$

Bokov A, Chaudhuri A, Richardson A (2004) The role of oxidative damage and stress in aging. Mech Ageing Dev 125:811-826

Brunk UT, Terman A (2002) Lipofuscin: mechanisms of agerelated accumulation and influence on cell function. Free Radic Biol Med 33:611-619

Calabrese V, Guagliano E, Sapienza M, Panebianco M, Calafato S, Puleo E, Pennisi G, Mancuso C, Butterfield DA, Stella AG (2007) Redox regulation of cellular stress response in aging and neurodegenerative disorders: role of vitagenes. Neurochem Res 32:757-773

Calabrese V, Cornelius C, Cuzzocrea S, Iavicoli I, Rizzarelli E, Calabrese EJ (2011) Hormesis, cellular stress response and vitagenes as critical determinants in aging and longevity. Mol Aspects Med 32:279-304 
Calabrese EJ, Dhawan G, Kapoor R, Iavicoli I, Calabrese V (2015) What is hormesis and its relevance to healthy aging and longevity. Biogerontology 16:693-707

Chavez V, Mohri-Shiomi A, Maadani A, Vega LA, Garsin DA (2007) Oxidative stress enzymes are required for DAF-16mediated immunity due to generation of reactive oxygen species by Caenorhabditis elegans. Genetics 176:1567-1577

Chen KJ (2015) Reflections on human longevity and Chinese medicine prevention and treatment of chronic diseases. Chin J Integr Med 21:643-647

Chen X, Zhang J, Fang Y, Zhao C, Zhu Y (2008) Ginsenoside $\operatorname{Rg} 1$ delays tert-butyl hydroperoxide-induced premature senescence in human WI-38 diploid fibroblast cells. J Gerontol A 63:253-264

Chen Q, Liu K, Robinson AR, Clauson CL, Blair HC, Robbins PD, Niedernhofer LJ, Ouyang H (2013) DNA damage drives accelerated bone aging via an NF-kappaB-dependent mechanism. J Bone Miner Res 28:1214-1228

Chen C, Mu XY, Zhou Y, Shun K, Geng S, Liu J, Wang JW, Chen J, Li TY, Wang YP (2014) Ginsenoside Rg1 enhances the resistance of hematopoietic stem/progenitor cells to radiation-induced aging in mice. Acta Pharmacol Sin 35:143-150

Cheng Y, Shen LH, Zhang JT (2005) Anti-amnestic and antiaging effects of ginsenoside $\operatorname{Rg} 1$ and $\mathrm{Rb} 1$ and its mechanism of action. Acta Pharmacol Sin 26:143-149

Chung HY, Lee EK, Choi YJ, Kim JM, Kim DH, Zou Y, Kim CH, Lee J, Kim HS, Kim ND, Jung JH, Yu BP (2011) Molecular inflammation as an underlying mechanism of the aging process and age-related diseases. J Dent Res 90:830-840

Finkel T, Holbrook NJ (2000) Oxidants, oxidative stress and the biology of ageing. Nature 408:239-247

Gladyshev TV, Gladyshev VN (2016) A disease or not a disease? Aging as a pathology. Trends Mol Med 22:995-996

Guarente L, Kenyon C (2000) Genetic pathways that regulate ageing in model organisms. Nature 408:255-262

Honda Y, Tanaka M, Honda S (2010) Redox regulation, gene expression and longevity. Geriatr Gerontol Int 10(Suppl 1):S59-S69

Hsu YC, Chiu CJ, Wray LA, Beverly EA, Tseng SP (2015) Impact of traditional Chinese medicine on age trajectories of health: evidence from the Taiwan Longitudinal Study on Aging. J Am Geriatr Soc 63:351-357

Hu GR (1986) T cell function in aged persons treated with a kidney-reinforcing principle. Zhong Xi Yi Jie He Za Zhi 6(264-267):258

Isobe KI (2016) Mechanism of immune aging. Nihon Rinsho 74:1497-1501

Kitani K, Minami C, Isobe K, Maehara K, Kanai S, Ivy GO, Carrillo MC (2002) Why (-)deprenyl prolongs survivals of experimental animals: increase of anti-oxidant enzymes in brain and other body tissues as well as mobilization of various humoral factors may lead to systemic anti-aging effects. Mech Ageing Dev 123:1087-1100

Ko KM, Chiu PY, Leung HY, Siu AH, Chen N, Leong EP, Poon MK (2010) Long-term dietary supplementation with a yang-invigorating Chinese herbal formula increases lifespan and mitigates age-associated declines in mitochondrial antioxidant status and functional ability of various tissues in male and female C57BL/6J mice. Rejuvenation Res 13:168-171

Krut'ko VN, Dontsov VI, Khalyavkin AV, Markova AM (2018) Natural aging as as a sequential poly-systemic syndrome. Front Biosci 23:909-920

Lee JH, Choi SH, Kwon OS, Shin TJ, Lee JH, Lee BH, Yoon IS, Pyo MK, Rhim H, Lim YH, Shim YH, Ahn JY, Kim HC, Chitwood DJ, Lee SM, Nah SY (2007) Effects of ginsenosides, active ingredients of Panax ginseng, on development, growth, and life span of Caenorhabditis elegans. Biol Pharm Bull 30:2126-2134

Li J, Cai D, Yao X, Zhang Y, Chen L, Jing P, Wang L, Wang Y (2016) Protective effect of ginsenoside $\mathrm{Rg} 1$ on hematopoietic stem/progenitor cells through attenuating oxidative stress and the Wnt/beta-catenin signaling pathway in a mouse model of D-galactose-induced Aging. Int $\mathbf{J}$ Mol Sci 17:249

Liang ZH, Yin DZ (2010) Preventive treatment of traditional Chinese medicine as antistress and antiaging strategy. Rejuvenation Res 13:248-252

Liu J, Peng L, Huang W, Li Z, Pan J, Sang L, Lu S, Zhang J, Li W, Luo Y (2017) Balancing between aging and cancer: molecular genetics meets traditional Chinese medicine. J Cell Biochem 118:2581-2586

Mattson MP (2008) Hormesis and disease resistance: activation of cellular stress response pathways. Hum Exp Toxicol 27:155-162

Mikhelson VM, Gamaley IA (2012) Telomere shortening is a sole mechanism of aging in mammals. Curr Aging Sci 5:203-208

Mukhopadhyay A, Oh SW, Tissenbaum HA (2006) Worming pathways to and from DAF-16/FOXO. Exp Gerontol 41:928-934

Munoz MJ, Riddle DL (2003) Positive selection of Caenorhabditis elegans mutants with increased stress resistance and longevity. Genetics 163:171-180

Murphy CT, McCarroll SA, Bargmann CI, Fraser A, Kamath RS, Ahringer J, Li H, Kenyon C (2003) Genes that act downstream of DAF-16 to influence the lifespan of Caenorhabditis elegans. Nature 424:277-283

Seo HW, Cheon SM, Lee MH, Kim HJ, Jeon H, Cha DS (2015) Catalpol modulates lifespan via DAF-16/FOXO and SKN1/Nrf2 activation in Caenorhabditis elegans. Evid Based Complement Altern Med 2015:524878

Shen ZY, Zheng Z, Guo WM (2002) Clinical and experimental study on retardation of immunosenescence by kidney tonifying principle. Zhongguo Zhong Xi Yi Jie He Za Zhi 22:178-181

Sluder AE, Baumeister R (2004) From genes to drugs: target validation in Caenorhabditis elegans. Drug Discov Today Technol 1:171-177

Tang YL, Zhou Y, Wang YP, Wang JW, Ding JC (2015) SIRT6/ NF-kappaB signaling axis in ginsenoside Rg1-delayed hematopoietic stem/progenitor cell senescence. Int J Clin Exp Pathol 8:5591-5596

Wan F, Zhi D, Liu D, Xian J, Li M, AbuLizi A, Ju W, Li H (2014) Lifespan extension in Caenorhabiditis elegans by several traditional Chinese medicine formulas. Biogerontology 15:377-387

Wang Q, Yang F, Guo W, Zhang J, Xiao L, Li H, Jia W, Huang Z (2014) Caenorhabditis elegans in Chinese medicinal 
studies: making the case for aging and neurodegeneration. Rejuvenation Res 17:205-208

Yang Y, Ren C, Zhang Y, Wu X (2017) Ginseng: an nonnegligible natural remedy for healthy aging. Aging Dis 8:708-720

Zhang X, Zhang A, Jiang B, Bao Y, Wang J, An L (2008) Further pharmacological evidence of the neuroprotective effect of catalpol from Rehmannia glutinosa. Phytomedicine 15:484-490

Zhang X, Jin C, Li Y, Guan S, Han F, Zhang S (2013) Catalpol improves cholinergic function and reduces inflammatory cytokines in the senescent mice induced by D-galactose. Food Chem Toxicol 58:50-55

Zhao L, Zhao Y, Liu R, Zheng X, Zhang M, Guo H, Zhang H, Ren F (2017) The transcription factor DAF-16 is essential for increased longevity in C. elegans exposed to Bifidobacterium longum BB68. Sci Rep 7:7408

Zhou DH (1982) Preventive geriatrics: an overview from traditional Chinese medicine. Am J Chin Med 10:32-39

Zhou Y, Yang B, Yao X, Wang Y (2011) Experimental study of relationship between effect of ginsenoside $\operatorname{Rg} 1$ to delay hematopoietic stem cell senescence and expression of p16(INK4a). Zhongguo Zhong Yao Za Zhi 36:608-613

Zhou Y, Liu J, Cai S, Liu D, Jiang R, Wang Y (2015) Protective effects of ginsenoside $\operatorname{Rg} 1$ on aging Sca-1(+) hematopoietic cells. Mol Med Rep 12:3621-3628

Publisher's Note Springer Nature remains neutral with regard to jurisdictional claims in published maps and institutional affiliations. 